\title{
High frequency Modelling of Cables in PWM Motor Drives by Using Polynomial Functions based Parameters
}

\author{
M. C. Di Piazza, Member IEEE, A. Ragusa, Member IEEE, G. Vitale, Member IEEE \\ Consiglio Nazionale delle Ricerche, \\ Istituto di Studi sui Sistemi Intelligenti per l'Automazione, \\ (ISSIA - CNR), sezione di Palermo, Palermo, Italy, \\ mariacarmela.dipiazza@ieee.org; ragusa@ieee.org; gianpaolo.vitale@ieee.org
}

\begin{abstract}
The high frequency behaviour of the cables in pulse width modulation (PWM) motor drives is of a crucial importance in the analysis of transient overvoltages and conducted electromagnetic interference (EMI) propagation, both common and differential mode. In order to give a tool for simulation of the above cited phenomena, a new method for high frequency cable modelling is proposed in this paper. The method is based on the measurement of the impedance frequency responses of the cables in short and open circuit conditions. The lumped parameters cable behaviour is described by polynomial functions in frequency domain. For a given frequency a simple equivalent circuit representation of the cable is obtained. The proposed model is easily implemented in Matlab/Simulink and it is validated comparing the computed frequency impedance responses of the cable, loaded by either a resistive load or an induction motor, to the corresponding measured ones. Moreover, the prediction, by the model, of the overvoltage at the motor terminals in a long-cable PWM drive is performed. The proposed model are also compared with some commonly used high frequency cable models presented in technical literature.
\end{abstract}

\section{Keywords}

High frequency modelling, Parameters identification, Induction motor drives

\section{Introduction}

The high speed commutation in modern pulse width modulation (PWM) motor drives is responsible for very rapid voltages and currents transitions that lead to several serious problems ranging from conducted and radiated electromagnetic interference (EMI) to overvoltages, up to two or three times the DC link voltage, at the motor terminals when long cable configurations are used.

The overvoltage, determined by reflection phenomena, is tied to the impedance mismatch between the cable and the load represented by the motor. Its magnitude is dependent both on the cable length and characteristics and on the rise time of power devices pulses. In particular, the switching of power devices is a step solicitation that can excite a travelling wave from the inverter to the motor. Furthermore, the reflected wave, associated to the traveling wave, gives a contribution to the common mode (CM) EMI affecting the reliability of the motors since they produce shaft voltages and possible bearing currents [1]. For all these reasons the development of accurate high frequency cable simulation models is crucial for an appropriate analysis of overvoltages and EMI in power drive systems. Several contributions based on lumped parameters schematization of the cables have been presented in technical literature [2]-[6]. A model based on mathematical formulas describing the transient voltage and current in the cable in the frequency domain is given in [7]. It permits to analyze the overvoltage at any point of the cable without the need to give a circuit representation of the drive components, anyway it requires a detailed and noteworthy analytical formulation of the propagation phenomena.

The cable representation by several lumped parameters equivalent sections has been successfully proposed [2][4]. In such methods the model parameters identification is based on the impedance measurement. The appropriate choice of the sections' number is the key issue in this case since a tradeoff solution is needed to conjugate accuracy and fast simulation times.

In [2] the multiple lumped parameters segments approach is used to develop a high frequency model of the cable in a PWM drive, where a lossy representation of the line is proposed. In this paper the frequency dependency of the cable parameters is accounted for by measuring the impedance frequency responses at only two frequencies, i.e., the lowest and the highest of the considered test range.

In [3] a ladder-type network is proposed in order to model the cables including the frequency dependence of their parameters and the variations due to the skin effect and proximity. This model, indicated as "n-branch" gives accurate results to represent the behavior of the cable longitudinal parameters but it needs an appropriate modification of the original structure to model the transversal ones.

In this paper the proposed method utilizes experimental measurements in order to achieve short and open circuit impedance of the cable. For a fixed frequency value, in 
the range between $100 \mathrm{kHz}$ and $2 \mathrm{MHz}$, a simple equivalent circuit representation of the cable is obtained, consisting of a longitudinal inductive-resistive branch and two transversal capacitive branches with constant parameters. The model is easily implemented in Matlab/Simulink and is validated comparing the computed frequency impedance responses of the cable to the corresponding measured ones. The obtained model reproduces the overvoltage phenomena over the frequency spectrum of the voltage pulse. The obtained results, in terms of impedance response, are compared with different commonly used high frequency cable models presented in technical literature.

\section{Transient Motor Overvoltage in PWM Drives}

The presence of the power cables between the inverter and the motor introduces a natural oscillation frequency and a damping factor in the propagating waves.

The oscillation is excited by a voltage step variation and depends on the cable length while the damping factor is tied to the cable lossy behaviour.

The main factors involved in the generation of motor overvoltage are: the cable characteristics, the cable to motor surge impedance mismatch and the rise time of the PWM pulses [1], [8]. In a word, the peak transient voltage at the motor is caused by the reflected wave resulting by the cable to motor impedance mismatch and to the extremely small rise times of the voltage pulse. In particular if $Z_{m}$ represents the motor impedance and $Z_{c}$ the cable characteristic impedance, the peak value of the reflected wave voltage, $V_{m p}$ is given by:

$$
V_{m p}=V_{D C}(1+\gamma)
$$

where $\gamma=\left(Z_{m}-Z_{c}\right) /\left(Z_{m}+Z_{c}\right)$ gives a measure of the impedance mismatch and $V_{D C}$ is the DC link bus voltage. Transient overvoltages at ac motors terminals, due to the inverter commutation-motor-cable dynamic interaction, have become more and more heavy as the switching speed of the power semiconductors has increased from gate turn off (GTO) devices to currently used IGBT's.

The modern IGBT technology has determined a strong reduction of rise times (in the range from 50 to $400 \mathrm{~ns}$ ), leading to overvoltages equal to $2 V_{D C}$ at very short cable lengths, even shorter than $10 \mathrm{~m}$. Indicating with $\tau_{r}$ the rise time of the PWM pulse, the critical length of the cable (corresponding to a motor reflected peak voltage of $\left.2 V_{D C}\right), \ell_{c}$, is determined as:

$$
\ell_{c}=\frac{1}{4}\left(c / \sqrt{\varepsilon_{r}}\right)\left(\pi \tau_{r}\right)
$$

where $c$ is the speed of light and $\varepsilon_{r}$ is the relative dielectric constant of the cable. Moreover, the use of PWM with high carrier frequencies in association to cable length higher than the critical one worsens the problem, leading to overvoltages up to $3 V_{D C}$. This phenomenon is related to a complex interaction of repeating pulses and cable behavior not permitting the transient reflected wave to decay to zero before the application of the next pulse, so that a residual cable charge condition occurs.

\section{The proposed model}

The presented method is based on the capture of the most important electrical parameters by measurements performed on the cable under test. In particular, two types of measurements are carried out. The first is the short-circuit measurement, which allows the determination of the line impedance versus frequency $Z_{s h}(f)$ and the phase angle versus frequency $\phi_{s h}(f)$. In this way, from the $Z_{s h}(f)$ and $\phi_{s h}(f)$ diagrams, the longitudinal line parameters, i.e,. resistance versus frequency $R(f)$ and inductance versus frequency $L(f)$, are obtained. Similarly, the open-circuit measurement allows the determination of the line impedance versus frequency $Z_{o c}(f)$ and the phase angle versus frequency $\phi_{o c}(f)$. In this way, from the $Z_{o c}(f)$ and $\phi_{o c}(f)$ diagrams, the transversal line parameter, i.e., the capacitance versus frequency $C(f)$ is obtained. The measurements carried out are concerning the particular line with length $l$. From the obtained diagrams $R(f), L(f)$, and $C(f)$, it is possible to extract three polynomials $R_{p o l}(f)$, $L_{p o l}(f)$, and $C_{p o l}(f)$, suitably fitting the experimental data, by least squares methods. The three polynomials are then used in Simulink SimPowerSystem blocks to simulate the frequency dependence of the $R, L$ and $C$. Obviously for a complete investigation of the phenomena, measurements of the motor impedance vs. frequency are performed too, in order to characterize the entire system. The measured motor parameters are fitted by polynomials as well.

It is important to remark the relation between the physical length $l$ and the electrical length $\tilde{\lambda}$ of the line. When the physical length $l$ of a power line is comparable or shorter than the wavelength related to the higher frequency that occurs, the line is defined "electrically long" and it is necessary to study its electric quantities (voltage and current profiles) with a distributed parameters circuit. Nevertheless, it is also possible to study the cable electric behavior with a cascade of a proper number of "pi-cells" with resistances, inductances and capacitances, achieving a good approximation. In this paper the "pi-cells" approach is adopted. In Fig.1 the scheme of a single cell used in the proposed model is drawn. The empirical rule used to define the minimum number $N$ of "pi-cells" is:

$N \geq 5 l \sqrt{L_{u} C_{u}} / \tau$

where: $l$ is the physical length of the cable, in $\mathrm{m}, L_{u}$ and $C_{u}$ are the line inductance and capacitance per unit length and $\tau$ is the rise time of the fastest signal to be studied [4]. The prediction of the overvoltages at the motor terminals occurring when a PWM inverter is used, is shown in the following section. It should be noted that the cable behavior in time domain is linear and timeindependent. Thanks to this property, the superposition of the effects is possible and the model can be used for more complex waveforms with a large spectral content, as in 
the pulse-like waveforms generated by the PWM inverters. In particular, once the most significant frequencies of the pulse spectrum are recognized, for example by a Fast Fourier Transform, the overvoltage produced at the motor terminals can be evaluated summing the time responses to each sinusoidal components at those frequencies.

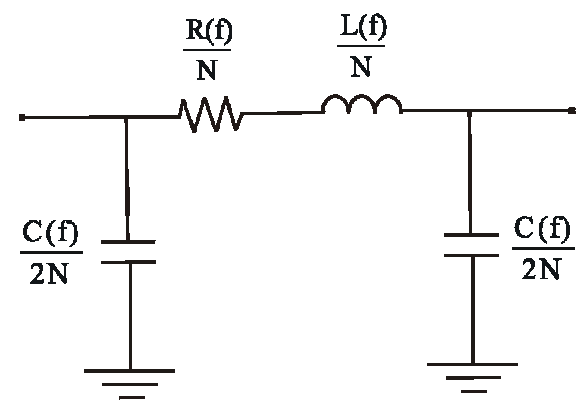

Fig.1. Scheme of the single cell of the proposed model.

\section{Experimental validation}

In order to obtain an experimental data set for the model validation the following test arrangement is used. A precision RLC meter Agilent 4285ALCR is used for the impedance vs. frequency measurements. The instrument is connected to a PC via a GPIB interface and it is automatically operated in a remote, controlled, and real time mode by using Labview software.

The frequencies of observation range from $200 \mathrm{kHz}$ to $2 \mathrm{MHz}$ with a resolution of $10 \mathrm{kHz}$. The line under study is composed of three not shielded unipolar cables with a section of $1.5 \mathrm{~mm}^{2}$ and a length of $10 \mathrm{~m}$. Two of the three cables are connected together in order to represent a bifilar line that is what the inverter sees in any configuration of its power devices. An open circuit measurement and a short circuit measurement are preformed in order to find out the transversal and longitudinal parameters of the line, respectively. In Fig. 2 the schemes of the measurement set up are shown. In Figs. $3 \mathrm{a}$ and $3 \mathrm{~b}$ the open circuit and short circuit cable measured impedance are reported, respectively.

As explained in the previous section, the open circuit measured impedance is used to identify the transversal line parameter, as a capacitance versus frequency $\mathrm{C}(\mathrm{f})$; on the contrary, the short circuit cable measured impedance is used to identify an equivalent longitudinal L-R series circuit. Figure 4 shows the obtained values of the capacitance, of the inductance and of the resistance versus frequency, respectively. Finally, the experimental results are used for the validation of the proposed model and for the implementation of other high frequency cable modeling methods. These methods are described in the following section in order to be compared with the proposed approach.

In Fig. 5 the impedance frequency responses of the cable loaded by a $50 \Omega$ resistive load, obtained by measurements and simulation, respectively, are shown.

In Fig. 6 the impedance frequency responses of the cable loaded by a $0.75 \mathrm{~kW}$ induction motor, obtained by measurements and simulation, respectively, are shown. It can be observed that the proposed model predicts the impedance vs. frequency behaviour up to $2 \mathrm{MHz}$ with a very good accuracy. As for the evaluation of the overvoltages at the motor terminals, the used experimental arrangement includes a digital oscilloscope Agilent MSO6104A and two high voltage differential probes Agilent N2772A. The phase to phase voltage at the inverter output and the corresponding voltage at the motor terminals are measured when a descending front occurs at the inverter output.

In Fig. 7 the measured waveforms are shown. An overvoltage of about $200 \mathrm{~V}\left(\approx \mathrm{V}_{\mathrm{DC}} / 3\right)$ and a damped oscillation are noted at the motor terminals respect to the inverter waveform.

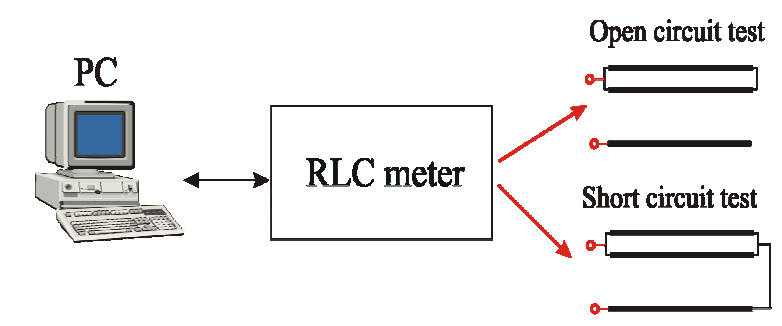

Fig.2. Scheme of the tests performed on the cable.
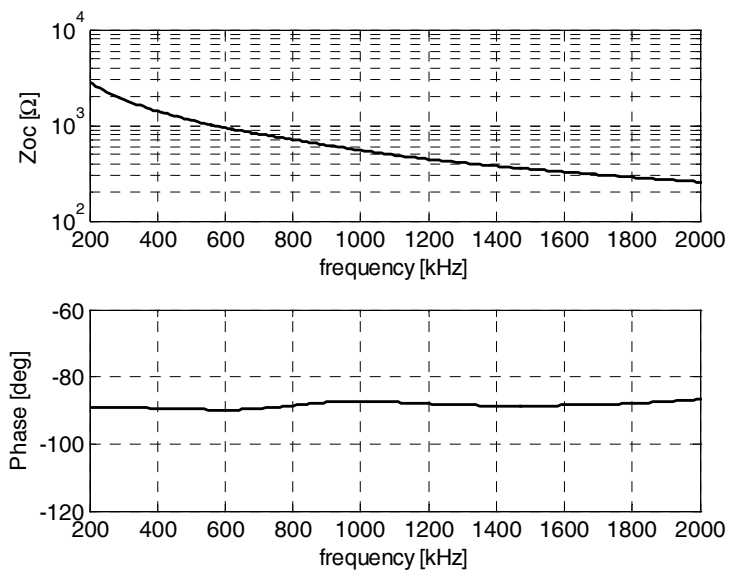

Fig. 3a. Open circuit cable measured impedance (amplitude and phase).
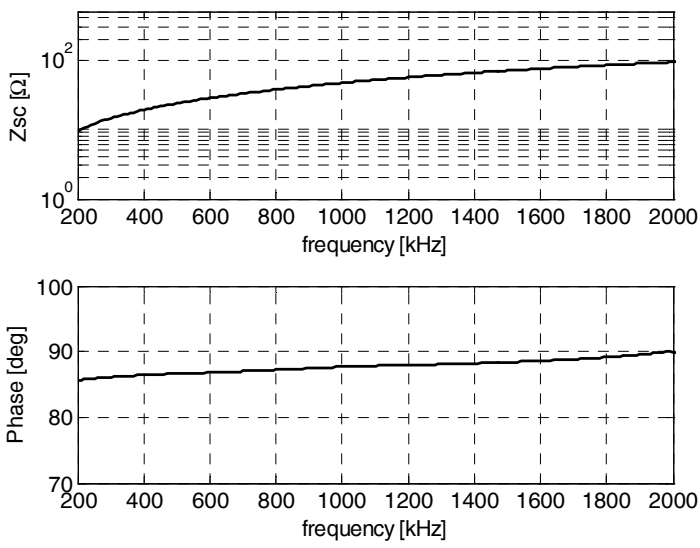

Fig. 3b. Short circuit cable measured impedance (amplitude and phase). 


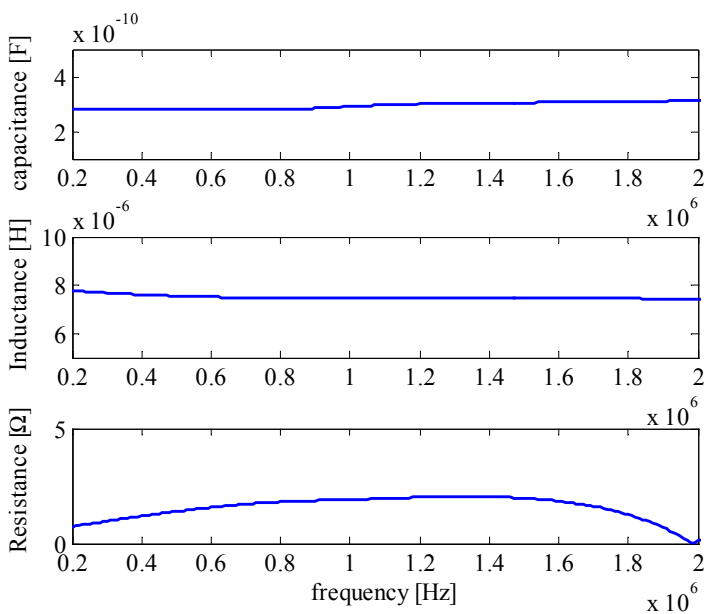

Fig.4. Experimental values of equivalent transversal capacitance (top), longitudinal inductance (middle) and resistance (bottom).

In order to test the model as a tool for the overvoltage prediction, a frequency decomposition of the inverter pulse is performed, by a Fast Fourier Transform algorithm. On the basis of the obtained spectrum, the first eight harmonics have been selected to approximate the pulse output by the inverter.

The complete response in time domain of the system composed of the studied cable and the induction motor is then obtained by adding each response to each selected frequency component of the input pulse. Fig. 8 shows the results obtained by simulation. It should be noted that, for the considered inverter output pulse, there are not significant harmonic components over $2 \mathrm{MHz}$.

In the considered frequency range the obtained values of $R(f), L(f)$ and $C(f)$ are quite constant, as shown in Fig. 4. Therefore the components of the scheme scketched in Fig. 1 can be set to the following constant values: $\mathrm{R}=2$ $\Omega, \mathrm{L}=7.5 \mu \mathrm{H}, \mathrm{C}=0.3 \mathrm{nF}$. In this case the pulse response can be obtained in the time domain.

The proposed model reproduces accurately the observed overvoltage at the motor terminals. Furthermore it allows to correctly estimates the delay propagation phenomena, indeed the delay of the voltage at the motor terminals respect to the inverter pulse is about $0.05 \mu \mathrm{s}$.

This value corresponds to the expected propagation speed $v=1 / \sqrt{L C}$ of $2 \cdot 10^{5} \mathrm{~km} / \mathrm{s}$, calculated for a lossless line by the transmission line theory [9].

\section{Comparison of the proposed model with other modelling methods}

Two different cable modeling methods are considered and compared with the proposed methodology. Both of the methods are able to describe transient overvoltage problems in long cable PWM motor drives reproducing the wave propagation and reflection phenomena.

The first method, called "n-branch" model, is based on a ladder-type network and it describes the cable parameters variation due to the skin effect and proximity. Considering the cable as an association of a finite shuntconnected concentric tubular sub-conductors, this method models the cable defining $n$-branches, each one with its own resistance, $R$, and inductance, $L$.

The $R-L$ elements do not vary with the frequency but they are suitably connected resulting in an equivalent impedance that reproduces the frequency variation of the cable parameters as shown in Fig. 9 (left). The values of the n-branches parameters are determined analytically from a set of cable resistance and inductance values, measured at different frequencies [3].

The $n$ pairs of these values are obtained by a shortcircuit impedance measurement over the studied frequency range. The number $n$ of branches is chosen in order to obtain the best representation of the cable configuration. The optimal solution is reached with the smallest number of branches that give an accurate representation of the $R$ and $L$ frequency variation in the studied frequency range.

The second method proposes a multiple segment lumped-parameter model for representing the cable. In Fig. 9 (right) the lumped parameter circuit per-unit length is shown. The parameters of the model are estimated analyzing the behavior of the short-circuit and opencircuit cable impedances [2]. Therefore, these two methods can be implemented using the same data set acquired for the proposed method. In particular, the cable model series parameters, $R_{s}$ and $L_{s}$, are obtained by the short-circuit impedance measurement, while the parallel parameters, $R_{p 1}, R_{p 2}, C_{p 1}$, and $C_{p 2}$, are associated to the open-circuit impedance measurement.

The parameters values are calculated using the shortcircuit and open circuit impedances values at only two frequencies, the lowest and the highest test frequencies. For this reason, this model will be referred in the following as the "2-freq" model, for the sake of simplicity. Both the "n-branch" and "2-freq" models are implemented in Matlab/Simulink. Both methods give results up to $2 \mathrm{MHz}$. The impedance response obtained with the proposed modeling method has been compared with those obtained by the two above described methods in the following configurations: a) cable terminated on a $50 \Omega$ resistive load; b) cable terminated on a $0.75 \mathrm{~kW}$ three phase induction motor. In Figs. 10 and 11 the comparison is shown for these two loads, respectively. From Figs. 10 and 11 it can be observed that the proposed method leads to the best prediction of the frequency impedance response. As a result, all studied methods require impedance measurements. The "nbranch" method gives frequency independent parameters but does not reproduces the transversal capacitive coupling among cables. The "2-freq" method gives frequency independent parameters as well but is based only on the knowledge of impedance a two frequencies. The method proposed in this paper needs of a complete set of measurements in the operating range of frequencies, on the other hand, it allows a simpler circuital representation to be determined. Moreover by a polynomial fitting, in general, the expression of parasitic parameters versus frequency can be obtained and, when these parameters are quite constant, a further simplification occurs and an elementary " $\Pi$ " cell described by a transmission matrix is easily obtained. 

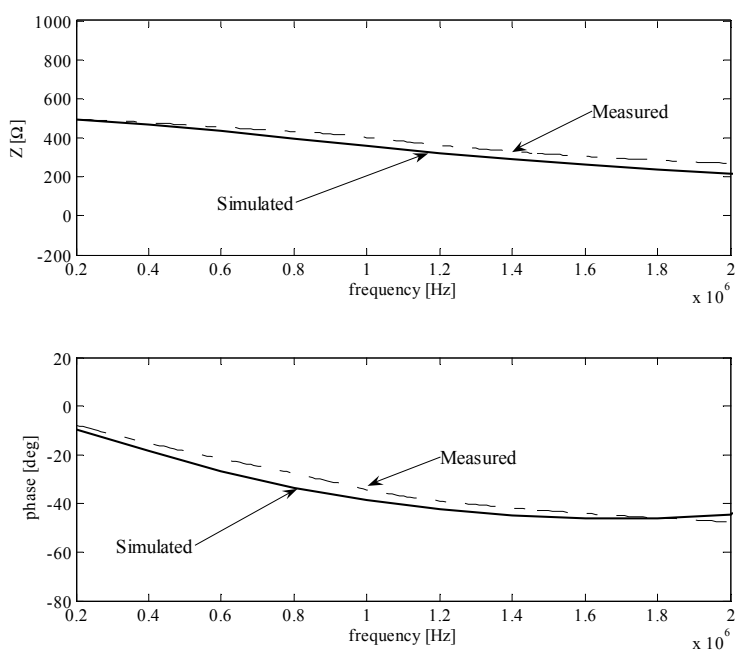

Fig.5. Measured and simulated impedance frequency responses of the cable loaded by a $50 \Omega$ resistive load.
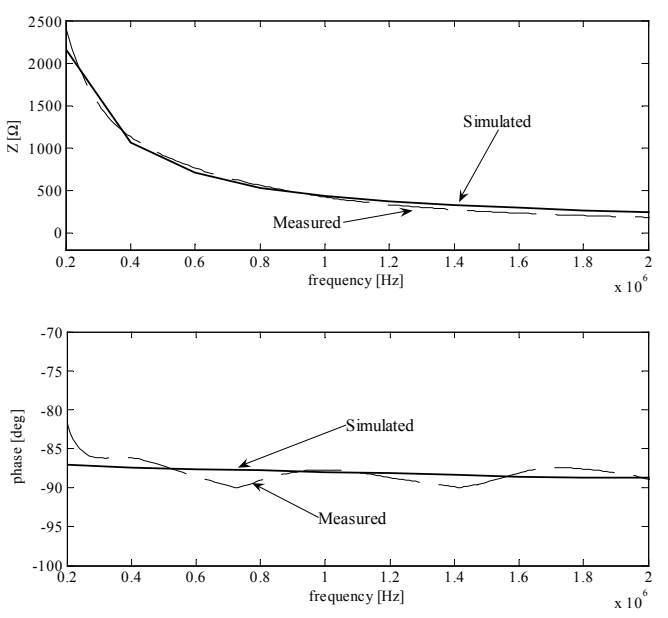

Fig.6. Measured and simulated impedance frequency responses of the cable loaded by a $0.75 \mathrm{~kW}$ induction motor.

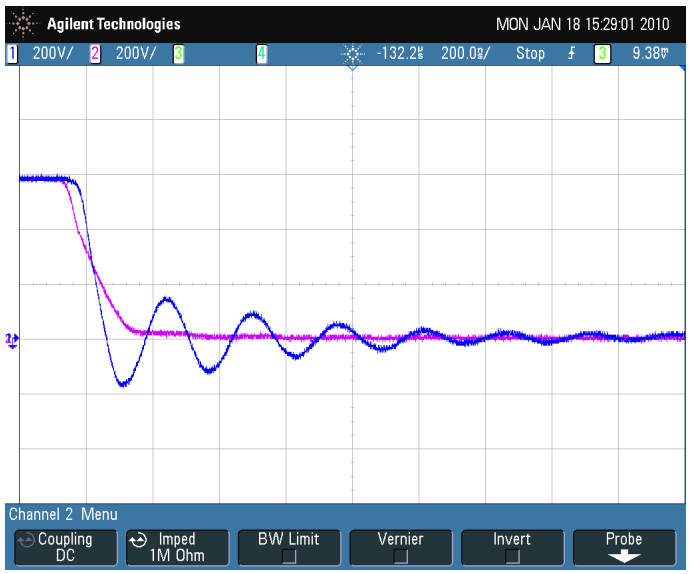

Fig.7. Measured inverter damped output voltage (purple trace) and oscillating voltage at the motor terminals (blue trace).

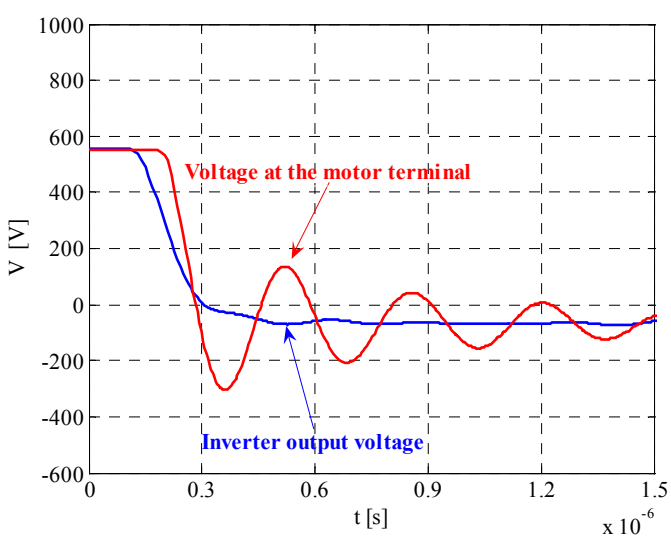

Fig.8. Simulated inverter output voltage and voltage at the motor terminals

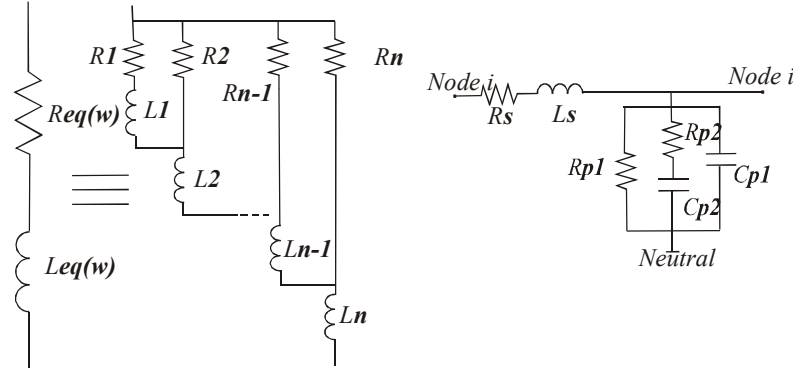

Fig. 9. N-branch circuit model (left) and 2-freq model per-unit length (right).
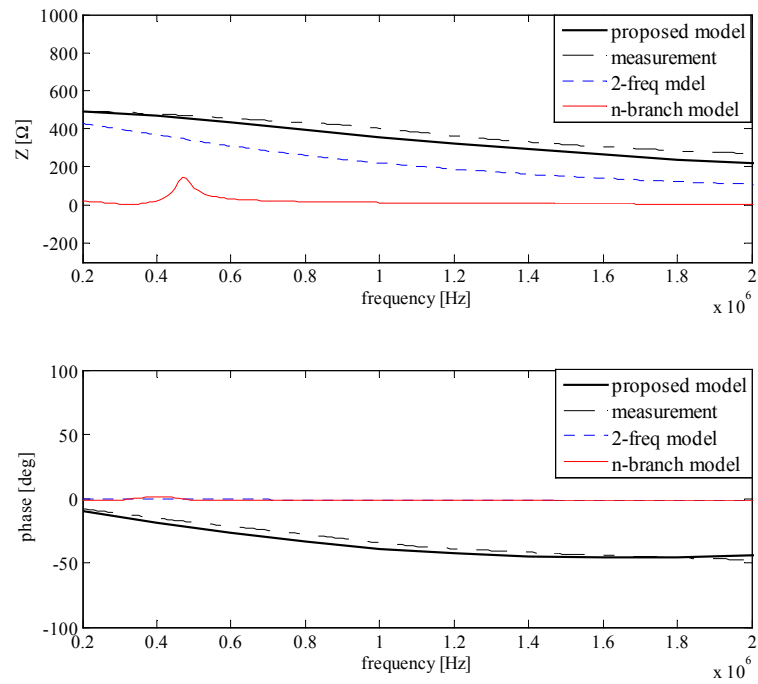

Fig.10. Comparison of the impedance frequency responses of the cable loaded by a $50 \Omega$ resistive load. 

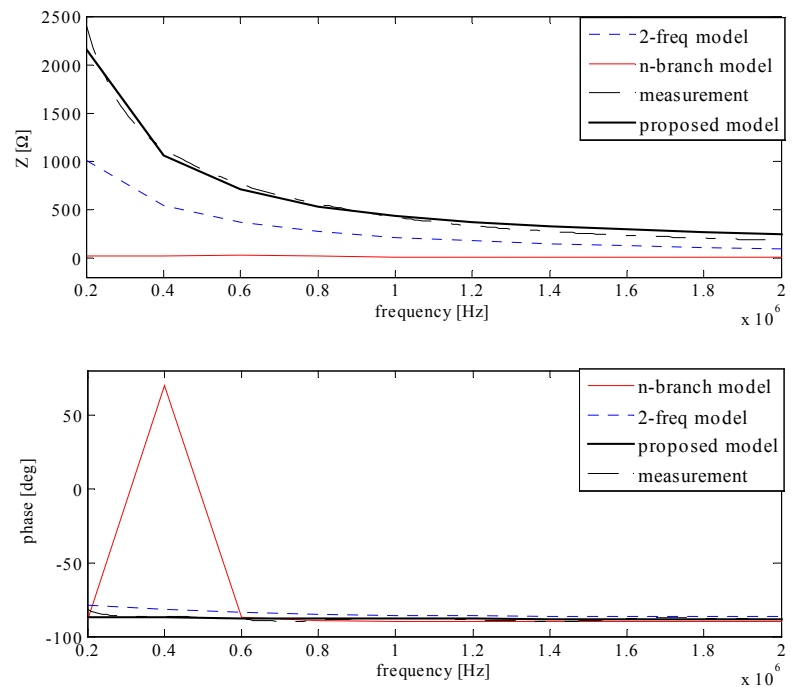

Fig.11. Comparison of the impedance frequency responses of the cable loaded by a $0.75 \mathrm{~kW}$ induction motor.

\section{Conclusions}

This paper presents a method for the high frequency modelling of the cables in PWM drives. It describes the cable behaviour by polynomial functions in frequency domain.

It requires impedance measurements and gives a simple equivalent circuit representation of the cable.

The proposed model allows transversal and longitudinal equivalent parameters to be determined versus frequency.

In general, it can be used in frequency domain by the superposition of the effects of each harmonic of the inverter pulse to predict overvoltages due to the impedance mismatch between the cable and the load represented by the motor. However, if the determined parameters are quite constant, a further simplification occurs and the model can be utilized directly in time domain.
In both cases an easily implementation in Matlab/Simulink allows the impedance responses of the cable to be reproduced and the overvoltage at the motor terminals in a long-cable PWM drive to be predicted. The comparison with other high frequency cable models, presented in technical literature, confirms the goodness of the proposed method.

\section{References}

[1] E. J. Bulington, S. Abney, G. L. Skibinski, "Cable alternatives for PWM AC drive applications", 46th annual Ind Applic. Soc. Petrol and chem. Ind.conf., Sept. 1999, pp.247-259.

[2] A. F. Moreira, T. A. Lipo, G. Venkataramanan, S. Bernet, "High-frequency modeling for cable and induction motor overvoltage studies in long cable drives", IEEE Trans. Ind. Appl., vol. 38, no. 5, pp. 1297-1306, Sept./Oct. 2002.

[3] H. De Paula, D. A. de Andrade, M. L. R. Chaves, J. L. Domingos, M. A. de Freitas, " Methodology for cable modeling and simulation for high-frequency phenomena studies in PWM motor drives", IEEE Trans. Power Electron., vol. 23, no.2, pp.744-752, March 2008.

[4] T. Dhaene, D. De Zutter, "Selection of lumped element models for coupled lossy transmission lines", IEEE Trans. on Computer-aided design, vol.11, no.7, July 1992, pp.805815. [5] L. Ran, S. Gokani, J.Clare, K.J. Bradley, C. Christopoulos, "Conducted electromagnetic emissions in induction motor drive systems part I:time domain analysis and identification of dominant modes", IEEE Trans. Power electron, vol. 13 no. 4, July 1998, pp.757-767.

[6] L. Ran, S. Gokani, J.Clare, K.J. Bradley, C. Christopoulos, "Conducted electromagnetic emissions in induction motor drive systems part II:frequency domain models", IEEE Trans. Power electron, vol. 13 no. 4, July 1998, pp.768-776.

[7] S. Amarir, K.Al-Haddad, "A modeling technique to analyse the impact of inverter supply voltage and cable length on industrial motor drives", IEEE Trans. power electron., vol.23, no.2 March 2008, pp.753-762. [8] R. J. Kerkman, D. Leggate, G. L. Skibinski, "Interaction of drive modulation and cable parameters on AC motor transients", IEEE Trans. Ind. Appl., vol.33, no.3, May-June 1997, pp.722-731.

[9] Ramo Winnery Van Duzer, "Fields and waves in communication electronics", John Wiley. 